\title{
Prehospital DNR Orders: What Do Physicians in Washington Know?
}

\author{
Maria J. Silveira, MD, MA, MPH, ${ }^{* \dagger}$ Rick A. Buell, ${ }^{\ddagger}$ and Richard A. Deyo, MD, MPH ${ }^{\mathcal{N}}$
}

See editorial comments by Dr. Joanne Lynn and Cria Gregory on pp 1502-1503.

OBJECTIVES: To assess whether physicians know of Washington State's prehospital do-not-resuscitate (DNR) policy, 6 years after its implementation.

DESIGN: Cross-sectional survey.

SETTING: Washington State, April 2001.

PARTICIPANTS: Four hundred seventy-one practicing physicians.

MEASUREMENTS: Multivariate logistic regression was used to determine relationships between physician and practice characteristics with knowledge of policies governing advance care planning.

RESULTS: Among respondents, 60\% did not know that Washington State requires an emergency medical service (EMS)-specific DNR order authored by a physician. Seventynine percent did not know that patient-authored advance directives apply only in hospitals and medical offices.

CONCLUSION: The findings in this study suggest that most physicians in Washington State lack knowledge about the documentation needed for EMS personnel to forgo prehospital attempts at cardiopulmonary resuscitation. Further study is needed to determine whether physician education or legislative change is necessary. J Am Geriatr Soc 51:1435-1438, 2003.

Key words: do not resuscitate (DNR) orders; emergency medical services (EMS); advance directives; prehospital guidelines; physician survey introduction

Ctates vary in their policies governing the use of written $\checkmark$ advance directives directing emergency medical service

From the ${ }^{\dagger}$ General Medicine, Bioethics Program, University of Michigan, Ann Arbor, Michigan; *VA Health Services Research and Development Service, VA Ann Arbor Healthcare System, Ann Arbor, Michigan; ${ }^{\ddagger}$ Office of Emergency Medical and Trauma Prevention, Washington Department of Health, Tacoma, Washington; ${ }^{\S}$ Departments of Medicine and Health Services and "Center for Cost and Outcomes Research, University of Washington, Seattle, Washington.

This study was supported by the Robert Wood Johnson Foundation Clinical Scholars Program. The opinions and conclusions are those of the authors, and may not represent those of the Robert Wood Johnson Foundation.

Address correspondence to Maria J. Silveira, MD, MA, MPH, 300 North Ingalls Building, Room 7C27, Ann Arbor, MI 48109.

E-mail: mariajs@umich.edu
(EMS) personnel. Washington State and 34 other states (AK, AZ, AR, CA, CO, CT, FL, GA, HI, ID, IN, KS, KY, LA, MD, MI, MT, NV, NH, NM, NY, NC, OH, OK, RI, SC, TN, TX, UT, VA, WA, WV, WI, and WY) have enacted "prehospital do-not-resuscitate" (DNR) or "out-of-hospital DNR" statutes to govern this practice. ${ }^{1-4}$ Physicians typically author prehospital DNR orders, which function as doctors' orders. These orders guide EMS personnel in determining whether to attempt cardiopulmonary resuscitation (CPR) in the event of cardiopulmonary arrest. The American College of Emergency Physicians, ${ }^{5}$ the American Heart Association, ${ }^{6}$ the National Association of State Emergency Medical Services Directors, ${ }^{7}$ and the National Association of Emergency Medical Service Physicians have endorsed prehospital DNR orders., ${ }^{7,8}$

In 1995, the Washington Department of Health established guidelines for prehospital DNR orders in response to an unfunded mandate from the state legislature. ${ }^{9}$ These guidelines provide for the physician of record to complete an EMS-No CPR directive on a specific form purchased from the Department of Health. The physician must sign the form. The signed EMS-No CPR form or an EMS-No CPR bracelet (also obtained from the Department of Health) must be visible for EMS personnel to withhold or withdraw attempts at CPR. In the absence of the EMS-No CPR directive or bracelet, EMS personnel are required to attempt CPR on persons in cardiopulmonary arrest unless a physician instructs them otherwise. ${ }^{4}$

Because of the key role physicians play in completing the EMS-No CPR directive in Washington, it was felt that it was important to explore physician knowledge about this and other matters regarding advance care planning. This study was designed to ask physicians whether they knew that an EMS-No CPR directive is needed for patients who do not wish prehospital attempts at CPR by EMS personnel, 6 years after its implementation by Washington State.

It was hypothesized that most physicians would be unaware of this requirement. The investigators felt that these results could inform others throughout the country who have implemented or are considering implementing prehospital DNR policies such as the one in Washington State.

\section{MATERIALS AND METHODS}

One thousand five hundred of the 2,777 physicians in Washington State listed in the American Medical Associa- 
Table 1. Correct Response by Question

Question

Correct Answer

No

$187 / 466(40)$

According to Washington law, should EMS personnel follow John's advance directive and withhold resuscitative efforts?

As far as you know, in Washington State, John's advance directive applies to which medical personnel?

As far as you know, in Washington State, John's advance directive applies in which settings?

As far as you know, is there an EMS-specific advance directive in Washington State?

Who should pronounce John dead and sign his death certificate?

\begin{tabular}{lc}
\multicolumn{1}{c}{ Correct Answer } & n/N Correct (\%) \\
No & $187 / 466(40)$ \\
Physicians & $185 / 465(40)$ \\
Hospital and medical offices & $97 / 468(21)$ \\
Yes & $189 / 467(40)$ \\
Primary care physician of record & $257 / 456(56)$
\end{tabular}

Note: The five questions listed above followed a vignette regarding a hypothetical patient, John, who has terminal prostate cancer, has authored an advance directive

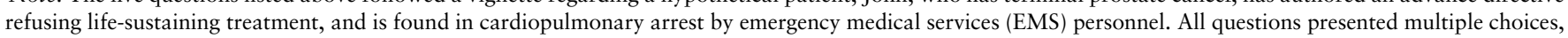

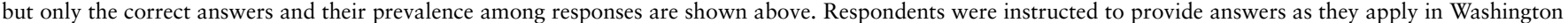
State according to state law.

tion (AMA) Physician Masterfile (AXCIOM, Skokie, IL) were surveyed. Physicians were eligible for the study if they had an active medical license, had a hospital-or office-based practice within Washington, and were younger than 60 (because they would be more likely to still practice medicine than older physicians who might maintain an active license despite retiring). Eligibility criteria were designed to help identify physicians likely to care for dying patients. Of the 2,777 physicians in Washington, 2,759 were eligible for this study.

From the population of 2,759 (99\%) eligible physicians, physicians were selected if they were randomly selected from among all eligible family practitioners $(1,599)$ and internists (947) or belonged to one of five specialties: critical care, cardiology, geriatrics, hematology/oncology, or infectious disease (212). The survey was mailed to 825 (52\% of eligible) randomly chosen family practitioners, $463(49 \%$ of eligible) randomly chosen primary care internists, and all $212(100 \%)$ eligible specialists. Specialists were oversampled (proportionally speaking) because of their relatively small numbers in the AMA Masterfile.

Physicians received an initial mailing of the survey in April including a cover letter from the Department of Health describing the purpose of the study and asking for their voluntary participation; 1,500 surveys were mailed in the initial mailing. A second mailing was sent to nonrespondents 2 weeks later. When questionnaires were returned undeliverable, attempts were made to identify a correct address using the Washington State Medical Association listing of physicians in the state. Respondents did not receive incentives for their participation. Questionnaires were tracked using a unique identifier to protect respondent confidentiality. All subjects were given an opportunity to request educational materials about the EMS-No CPR guidelines and advance directives through a postcard they could mail separately.

The questionnaire assessed physicians' knowledge of the EMS-No CPR guidelines and of advance directives in general. In addition, it asked physicians to describe their practice characteristics. Knowledge questions were in multiple choice or dichotomous format and followed a vignette of a hypothetical patient dying of cancer who has an advance directive and is found at home in cardiopulmonary arrest. The questions were designed to avoid leading and loaded language. Wherever possible, technical terms such as advance directives or prehospital DNR orders were defined or described. There were nine knowledge questions and 16 demographic questions.

The questionnaire was pilot tested on a convenience sample of 20 academic physicians and revised accordingly before broad distribution. The Washington Department of Health, Office of Emergency Medical and Trauma Prevention, reviewed the questionnaire and answer key for accuracy. The University of Washington institutional review board and Washington Department of Health approved the study and considered completion of the survey as implicit informed consent.

Data analysis included descriptive statistics for respondent characteristics and responses to knowledge questions. Bivariate analyses (chi-square and Student $t$ test analyses) and multiple logistic regression were used to examine physician factors hypothesized to be associated with knowledge. In these analyses, a binary knowledge score was used as the outcome of interest. This score was calculated by summing points for correct answers to five key knowledge questions (Table 1) then dichotomizing according to the median; correct answers were awarded 1 point, and incorrect or missing answers were given 0 points. Using this score as the outcome, the significance of characteristics that might associate with knowledge and could serve as markers for physicians needing education was tested. These characteristics included: care for the dying (yes/no), practice location (rural, small/medium city, and urban), year of residency graduation, practice type (patient care vs nonpatient care), and primary specialty (family practice, internal medicine, hematology/oncology, and other). All analyses were conducted using STATA/SE 7.0 Software (STATA Corporation, College Station, TX).

\section{RESULTS}

Of 1,500 physicians in the initial mailing, 471 returned a completed questionnaire, 52 had an undeliverable mailing address, and 18 were excluded (11 for not practicing in Washington and 7 for returning the questionnaire beyond 


\begin{tabular}{lc}
\hline \multicolumn{1}{c}{ Table 2. Respondent Characteristics } \\
\hline \multicolumn{1}{c}{ Characteristic } & $\mathrm{n}(\%)$ \\
\hline Primary professional activity $(\mathrm{n}=450)$ & \\
Office-based patient care & $370(82)$ \\
Hospital-based patient care & $47(10)$ \\
Research & $5(1)$ \\
Administration & $4(1)$ \\
Teaching & $4(1)$ \\
Other & $20(4)$ \\
Primary specialty $(\mathrm{n}=453)$ & \\
Family practice & $254(56)$ \\
Internal medicine & $124(27)$ \\
Hematology/oncology & $24(5)$ \\
Infectious disease & $14(3)$ \\
Geriatrics & $11(2)$ \\
Emergency medicine & $5(1)$ \\
Other & $21(5)$ \\
Practice setting ( $\mathrm{n}=452)$ & \\
Large urban & $275(61)$ \\
Small urban & $147(33)$ \\
Rural & $30(7)$ \\
Median years since residency $(\mathrm{n}=444)$ & $0-30(15)$ \\
Median years since fellowship $(\mathrm{n}=86)$ & $1-29(13)$ \\
\hline
\end{tabular}

the deadline), yielding a response rate of $33 \%$. Respondents' characteristics are summarized in Table 2.

Consistent with this selection criterion, the vast majority of respondents $(91 \%, 424 / 466)$ cared for dying patients in their clinical practice, although half saw two or fewer dying patients per month (range 0-100). Nearly all $(95 \%, 428 / 451)$ reported routinely encouraging patients to complete advance directives, with many $(79 \%, 356 / 451)$ making forms available for patients in their offices ( $82 \%$ of those caring for dying patients). Only a few $(11 \%, 47 / 427)$ routinely advised dying patients and their families how to obtain a death certificate.

Despite this reported experience in caring for dying patients, few physicians responded correctly to the survey questions (Table 2). The median knowledge score was 2 out of a possible 5 points (range $0-5)$. Only 33 respondents $(7 \%$ of 450 who responded to all five questions) answered all five questions correctly. In only one question did more than half the respondents answer correctly.

In bivariate analyses, respondents who scored above the median knowledge score were more likely to care for dying patients $(P=.005)$; practice in an urban setting $(P=.065)$; specialize in internal medicine, family practice, hematology/oncology, or geriatrics $(P=.035)$; and have more years since graduation (median 15 vs 14 years; $P=.057)$. When all personal and practice characteristics were explored simultaneously using multivariate logistic regression, only caring for dying patients predicted better knowledge (Table 3). After adjusting for other factors, physicians caring for dying patients were 2.5 times more likely to score above the median knowledge score than those who did not care for dying patients $(95 \%$ confidence interval $=1.2-5.3, P=.02)$.

Many respondents $(66 \%, 285 / 434)$ wanted additional information about advance directives, preferentially through written materials $(78 \%)$ rather than lectures $(16 \%)$ or other forms of communication.

In an area reserved for commentary, 80 respondents remarked on the survey or its subject matter. Most responders criticized current policy surrounding advance directives and CPR in Washington for being unnecessarily complex and burdensome for patients and providers. For example, one respondent wrote, "The current law is too cumbersome. Patients have to complete separate directives in hospital, with hospice, and upon entry to nursing homes. Can't we just do this once?!" Another wrote, "Having EMSspecific advance directives is too complicated. EMS personnel should be legally able to follow a person's legal advance directive." Three respondents recommended adopting physician's orders for life-sustaining treatment (POLST), a physician's directive currently used in Oregon. POLST are physician DNR orders that travel with an individual and are recognized by all healthcare providers in Oregon. ${ }^{10,11}$

\section{DISCUSSION}

This study found that many Washington State physicians were poorly informed about state policies regarding advance directives and prehospital DNR orders. Sixty

Table 3. Results of Multivariate Logistic Regression

\begin{tabular}{lccc}
\hline \multicolumn{1}{c}{ Characteristic } & Odds Ratio & $P$-value & 95\% Confidence Interval \\
\hline Care for dying patients & 2.5 & .02 & $1.2-5.3$ \\
Years since graduation (per 10 years since graduation) & 1.3 & .09 & $1.0-1.6$ \\
Primarily care for patients & 2.8 & .35 & $0.3-24.7$ \\
Practice location & - & & \\
$\quad$ Rural & 2.1 & .09 & $0.88-5.04$ \\
$\quad$ Small/medium city & 1.7 & .24 & $0.71-3.88$ \\
$\quad$ Urban & - & & \\
Primary specialty & 1.4 & .29 & $0.74-2.68$ \\
$\quad$ Others & 1.5 & .22 & $0.77-3.10$ \\
Family practice & 1.9 & .24 & $0.66-5.45$ \\
Internal medicine & & \\
Hematology/oncology & & & \\
\hline
\end{tabular}

Note: Multivariate model included all the characteristics listed above. The outcome of interest was bivariate knowledge score. 
percent did not know that prehospital DNR orders existed in Washington State (a percentage that reflects performance no better than random guessing) or that advance directives applied to physicians only and not emergency medical service personnel.

This raises concerns that physician ignorance of the EMS-No CPR guidelines would severely limit their success, because physicians are central in the process by which patients obtain prehospital DNR orders. The American Bar Association Commission on Legal Problems of the Elderly expressed similar misgivings after conducting a national survey of EMS directors regarding prehospital DNR protocols in 1999, in which virtually all states acknowledged that public and professional education concerning the protocols was "a major challenge, made worse by the lack of adequate resources to carry out effective education."7 Indeed, few states have allocated the necessary resources to promote prehospital DNR policies, ${ }^{7}$ but to determine these policies' effects on the number of unwanted resuscitations, more research must be done to measure their outcomes directly. Nonetheless, the present findings underscore the potential importance of educating physicians when such policies are implemented.

What might an educational program for doctors about prehospital DNR orders look like? These findings raise several points that should be considered in designing a response. First, knowledge about advance care planning appears to be related to experience. Physicians who routinely care for dying patients know more than those physicians who do not (although their knowledge is far from complete according to these findings). Physicians with more years in practice appear to be more knowledgeable than more-recent residency graduates. Therefore, educational campaigns might consider targeting physicians in training, when they are receptive to education and readily accessible through a central location. Alternatively, or in addition, education could occur as part of advanced cardiac life support or basic life support certification. Second, 18\% of physicians caring for dying patients still do not have ready access to advance directives and prehospital DNR forms. Publicity campaigns should aim to ensure physicians and patients know where to find such information (e.g., Websites).

An alternative conclusion is that prehospital DNR policies are difficult to implement and that what is warranted is not more physician education but rather reevaluation of such policies altogether. Perhaps the requirement of physician signature on prehospital DNR orders, or the alternative of uniformly applicable DNR orders, should be reconsidered. Indeed, three states (KY, LA, and OK) already permit patients to author prehospital DNR orders without physician authorization.

The results of this study must be interpreted with some caution in light of the study's limitations. First, the response rate was low-perhaps because incentives were not offered to the survey population - potentially introducing bias, but response rates for physician surveys are often low, especially among primary care physicians regarding topics outside daily practice. Second, even though the survey was carefully designed to avoid misleading respondents, the questions may have elicited opinion rather than true knowledge.

\section{ACKNOWLEDGMENTS}

We wish to thank Dr. Richard O. Cummins for his encouragement and thoughtful review of our survey design and Dr. Susan Dorr Goold and Prof. Carl Schneider for their careful commentaries on our manuscript.

\section{REFERENCES}

1. Sachs GA, Miles SH, Levin RA. Limiting resuscitation. Emerging policy in the emergency medical system. Ann Intern Med 1991;114:151-154.

2. Health Care Surrogate Decision-Making Legislation. Washington, DC: American Bar Association Commission on Legal Problems of the Elderly, 2000.

3. Leon MD, Wilson EM. Development of a statewide protocol for the prehospital identification of DNR patients in Connecticut including new DNR regulations. Ann Emerg Med 1999;34:263-274.

4. EMS-No CPR Guidelines. Tacoma, WA: Department of Health, 1995.

5. National guidelines for statewide implementation of EMS "do not resuscitate" (DNR) programs. National Association of State Emergency Medical Services Directors (NASEMSD) and the National Association of Emergency Medical Services Physicians (NAEMSP). Prehospital Disaster Med 1994;9:137-139.

6. Emergency Cardiac Care Committee and Subcommittees AHA. Guidelines for CPR and emergency cardiac care, Part 8: Ethical considerations in resuscitation. JAMA 1992;268:2282-2288.

7. Sabatino CP. Survey of state EMS-DNR laws and protocols. J Law Med Ethics 1999;27:294,297-315.

8. Sachs GA, Miles SH, Levin RA. Emergencies and advance directives. Hastings Cent Rep 1990;20:42-43.

9. Amendments to the Natural Death Act. 43, Vol. 70, Rev. Code Wash. (ARCW) $\S 70.122 .043$ (1992), Title 70. Public Health and Safety, Chapter 70.122. Natural Death Act, $\S 70.122 .043$. Annotated Revised Code of Washington, 1992.

10. Tolle SW, Tilden VP, Nelson CA et al. A prospective study of the efficacy of the physician order form for life-sustaining treatment [see comments]. J Am Geriatr Soc 1998;46:1097-1102.

11. Lee MA, Brummel-Smith K, Meyer J et al. Physician orders for life-sustaining treatment (POLST): Outcomes in a PACE program. Program of all-inclusive care for the elderly. J Am Geriatr Soc 2000;48:1219-1225. 